\title{
Genotypic characterisation and antimicrobial resistance of Pseudomonas aeruginosa strains isolated from patients of different hospitals and medical centres in Poland
}

\author{
Marcin Brzozowski , Żaneta Krukowska', Katarzyna Galant ${ }^{2}$, Joanna Jursa-Kulesza ${ }^{1}$ and Danuta Kosik-Bogacka ${ }^{3{ }^{*}}$ (D)
}

\begin{abstract}
Background: Pseudomonas aeruginosa is a Gram-negative bacteria responsible for infections in immunocompromised patients and is one of the most common causes of nosocomial infections particularly in intensive care and burn units. We aimed to investigate the population structure of $P$. aeruginosa strains isolated from patients at different hospital wards. Methods: We analysed the possible presence of $P$. aeruginosa epidemic or endemic strains in hospitals of the selected region. A genotyping analysis was performed for $P$. aeruginosa isolates $(n=202)$ collected from patients of eleven hospitals in north-western Poland. Collections of $P$. aeruginosa were genotyped using pulsed-field gel electrophoresis (PFGE). Phenotypic screening for antibiotic susceptibility was performed for the common antimicrobial agents.

Results: Pseudomonas aeruginosa isolates were distributed among 116 different pulsotype groups. We identified 30 groups of clonally related strains, each containing from 2 to 17 isolates and typed the obtained 13 unique patterns, designated as $A, D, E, J, K, M, N, O, P, T, X, A C, A D$, and $A H$. The two largest clusters, $D$ and $E$, contained 17 and 13 isolates, respectively. Strains of these groups were continuously isolated from patients at intensive care units and burn units, indicating transmission of these strains.

Conclusions: In this study, we demonstrate the clonal relatedness of $P$. aeruginosa strains and their constant exchange in hospitals over a period of 15 months. The obtained results indicate a predominantly non-clonal structure of $P$. aeruginosa.
\end{abstract}

Keywords: Pseudomonas aeruginosa, Genotyping, Antimicrobial resistance, PFGE, Population structure

\section{Background}

Pseudomonas aeruginosa is a Gram-negative opportunistic pathogen that causes nosocomial infections for patients with pre-existing lung disease, including cystic fibrosis or chronic obstructive pulmonary disease, or patients on mechanical ventilation in the intensive care (ICUs). Eradication of $P$. aeruginosa in hospitals is especially problematic due to its intrinsic resistance to many

\footnotetext{
* Correspondence: kodan@pum.edu.pl

${ }^{3}$ Independent of Pharmaceutical Botany, Pomeranian Medical University in Szczecin, Powstanców Wielkopolskich 72, 70-111 Szczecin, Poland Full list of author information is available at the end of the article
}

antibiotic classes and its capacity to acquire resistance to all effective antibiotics $[1,2]$. Pseudomonas aeruginosa colonization of patients may originate from different exogenous sources such as sanitary installations (sinks, hot tubs, showers, etc.), contaminated diagnostic devices, mechanical ventilation, and cleaning equipment [3]. Infections of healthy individuals are rather rare; however, healthy colonised patients can serve as a continuous source of $P$. aeruginosa transmission [4].

Pseudomonas aeruginosa outbreaks occur in ICUs, neonatal ICUs, burn units (BU), haematological units, and other hospital wards where immunocompromised 
or critically ill patients are treated [5-8]. ICU patients are particularly at risk of $P$. aeruginosa infections due to the length of their stay in the medical ward, the severity of their illness and exposure to invasive medical procedures. It has been reported that $P$. aeruginosa infections of ICU patients primarily manifest as acute lung infections [6]. According to data from the European Centre for Disease Prevention and Control (ECDC), $P$. aeruginosa is the most common cause of respiratory pneumonia and the third most frequent agent causing urinary tract infections at European ICUs [9]. Among the distinguished causes of $P$. aeruginosa infections, the authors highlight the main role of inappropriate disinfection rather than resistance to the disinfectant used [10, 11].

According to previous reports, $P$. aeruginosa has a non-clonal population structure [12]. It has been established that the diversity of $P$. aeruginosa clones in extant populations is mostly generated by a frequent recombination of strains $[12,13]$. This mechanism of undergoing recombination also significantly contributes to the continuous evolution of $P$. aeruginosa within the lungs of cystic fibrosis patients [14]. Despite the non-clonal population, it is possible to distinguish endemic, epidemic or even pandemic $P$. aeruginosa strains disseminating in hospitals $[12,15$, 16]. Despite the intrinsic resistance to several antimicrobials, $P$. aeruginosa may acquire additional resistance mechanisms to all routinely used antipseudomonal drugs [1]. Multidrug resistance to antimicrobial agents of strains responsible for nosocomial outbreaks have been frequently noted by the authors in previous investigations [17-19]. The increased antimicrobial resistance of $P$. aeruginosa strains is contributing to a higher mortality rate of infected patients, longer hospitalization, more severe illness, and higher costs of treatment [1].

In this study, we describe the population structure and antimicrobial resistance rates of $P$. aeruginosa strains isolated from patients in different medical units of hospitals in Poland. The aim of this study was to establish the population structure, presence and distribution of possible epidemic and endemic $P$. aeruginosa strains among patients of hospitals. We analysed antimicrobial resistance rates of selected strains. The results provided by this study did not reveal the presence of an outbreak, although some strains were acknowledged as endemic due to their prolonged isolation in hospitals.

\section{Methods}

\section{Bacterial isolates}

Pseudomonas aeruginosa isolates $(n=742)$ were collected from 541 patients of 11 different hospitals and medical centres located in north-western Poland between December 2015 and March 2017. From the first isolate per patient, 202 were randomly selected for typing by Pulsed Field Gel Electrophoresis and antimicrobial drug resistance analysis.

Hospitals where the isolation was carried out were designated with letters $\mathrm{H} 1-\mathrm{H} 11$. The largest number of selected isolates $(n=174)$ originated from four major general hospitals $\mathrm{H} 1-\mathrm{H} 4$, located in three different cities of north-western Poland. The isolation of $P$. aeruginosa strains was less frequent in other smaller specialised hospitals and care facilities in the chosen region of Poland and thus the number of strains from these medical units is lower. Detailed information regarding the hospitals and the amount of strains collected at different hospital wards is included in Table 1 . The selected strains were isolated from blood $(n=35)$, the lower respiratory tract $(n=76)$, wounds $(n=65)$, urine $(n=12)$, and ear and eye swab samples $(n=14)$. Firstly, $P$. aeruginosa isolates were identified in the hospitals' microbiology laboratories. Further genus identification was performed using the PCR method described by Spilker et al. [20].

\section{Antimicrobial susceptibility testing}

The antipseudomonal drugs assessed in the study included carbapenems (meropenem, imipenem), cephalosporins (ceftazidime, cefepime), aminoglycosides gentamicin, amikacin, tobramycin, fluoroquinolone (ciprofloxacin), a penicillin $+\beta$-lactamase inhibitor (piperacillin-tazobactam) and polymyxin (colistin). To identify antimicrobial susceptibility, the disk diffusion method was performed. Pseudomonas aeruginosa ATCC 27853 was used as quality control. Minimal Inhibitory Concentration (MIC) of colistin was determined with a broth microdilution-based method (ComASP ${ }^{\mathrm{mi}}$ Colistin test, Liofilchem) as the disk diffusion method is no longer recommended to evaluate polymyxins susceptibility. Concentrations used varied between $0.25 \mu \mathrm{g} / \mathrm{ml}$ and $16 \mu \mathrm{g} / \mathrm{ml}$. Tests were performed and interpreted according to the EUCAST guidelines [21].

\section{DNA isolation}

The chromosomal $P$. aeruginosa DNA was isolated with the CHEF Bacterial Genomic DNA Plug Kit (Bio-Rad Laboratories) with some modifications of the manufacturer's instructions. Pseudomonas aeruginosa isolates were cultured overnight at $37^{\circ} \mathrm{C}$ on cetrimide agar. In the first stage of isolation, bacterial suspensions in PBS equivalent to 4-4.5 McFarland were prepared, then incubated in a water bath at $37^{\circ} \mathrm{C}$ for $20 \mathrm{~min}$. A $100 \mu \mathrm{l}$ sample of each bacterial suspension was mixed with $100 \mu \mathrm{l}$ of melted $2 \%$ agarose gel at $50{ }^{\circ} \mathrm{C}$. These mixtures were allowed to solidify in plug moulds. The resulting agarose plugs were mixed by inversion with a proteinase buffer with $20 \mu \mathrm{l}$ of proteinase (CHEF Bacterial Genomic 
Table 1 Sample collection locations and number of isolates collected from examined wards (ICU, intensive care unit; BU, burn unit; SU, surgical unit; OU, other units; n, number of isolates; H1, Multispeciality Voivodeship Hospital in Gorzów Wielkopolski; H2, Regional Specialist Hospital in Gryfice; H3, Independent Public Clinical Hospital no. 1, Pomeranian Medical University in Szczecin; H4, Independent Public Clinical Hospital no. 2, Pomeranian Medical University in Szczecin; H5, Specialised Zdroje Hospital in Szczecin; H6, Sokolowski Specialist Hospital in Szczecin; H7, Hospital of the Ministry of the Interior and Administration in Szczecin; H8, West Pomeranian Oncology Centre in Szczecin; H9, West Pomeranian Hospice for Children in Szczecin; H10, Independent Public Clinical Hospital no. 1, Pomeranian Medical University in Police; H11, Residential Health Care Centre in Resko)

\begin{tabular}{|c|c|c|c|c|c|c|c|}
\hline \multirow[t]{2}{*}{ Hospital } & \multirow[t]{2}{*}{ City } & \multirow[t]{2}{*}{ Hospital type } & \multicolumn{5}{|c|}{ Number of isolates collected from medical units of different types } \\
\hline & & & ICU & BU & SU & OU & All units \\
\hline $\mathrm{H} 1$ & Gorzów Wlkp. & General hospital & 9 & 0 & 15 & 15 & 39 \\
\hline $\mathrm{H} 2$ & Gryfice & General hospital & 18 & 32 & 8 & 5 & 63 \\
\hline H3 & Szczecin & General hospital & 35 & 0 & 2 & 11 & 48 \\
\hline $\mathrm{H} 4$ & Szczecin & General hospital & 16 & 0 & 4 & 4 & 24 \\
\hline H5 & Szczecin & Specialised hospital & 1 & 3 & 0 & 2 & 6 \\
\hline H6 & Szczecin & Specialised hospital & 0 & 0 & 2 & 4 & 6 \\
\hline $\mathrm{H} 7$ & Szczecin & Specialised hospital & 1 & 0 & 2 & 2 & 5 \\
\hline H8 & Szczecin & Clinic & 0 & 0 & 1 & 1 & 2 \\
\hline H9 & Szczecin & Care facility & 0 & 0 & 0 & 1 & 1 \\
\hline $\mathrm{H} 10$ & Police & Branch of hospital $\mathrm{H} 3$ & 1 & 0 & 1 & 4 & 6 \\
\hline $\mathrm{H} 11$ & Resko & Care facility & 0 & 0 & 0 & 2 & 2 \\
\hline
\end{tabular}

DNA Plug Kit, Bio-Rad Laboratories), then incubated for $20 \mathrm{~h}$ at $50{ }^{\circ} \mathrm{C}$. After incubation, the proteinase solution was removed and the plugs washed three times with 1 $\mathrm{ml}$ of $1 \mathrm{x}$ wash buffer from the commercial kit (1 wash/1 h). The plugs were either stored at $4{ }^{\circ} \mathrm{C}$ or used immediately in further analyses.

\section{Restriction endonuclease digestion}

Before restriction digestion, plugs were washed once in a $0.1 \mathrm{x}$ wash buffer for $1 \mathrm{~h}$, followed by a subsequent wash with a tango buffer (Thermofisher), again for $1 \mathrm{~h}$. For the restriction endonuclease digestion, a SpeI restriction enzyme was used (Thermofisher). Each plug was resuspended in fresh tango buffer and $50 \mathrm{U}$ of SpeI was added, followed by incubation for $20 \mathrm{~h}$ at $37^{\circ} \mathrm{C}$.

\section{PFGE and data analysis}

One third of each plug was loaded into a $1.2 \%$ agarose, 0.5\% TBE gel. Pulsed-field gel electrophoresis (PFGE) was conducted with the CHEF-DRIII system (Bio-Rad Laboratories, USA), using $0.5 \mathrm{x}$ TBE as a running buffer and the following run conditions: $6 \mathrm{~V} / \mathrm{cm}, 14^{\circ} \mathrm{C}$, for $20 \mathrm{~h}$ with initial and final switch times 5 and $45 \mathrm{~s}$, respectively. Pseudomonas aeruginosa ATCC 27853 was applied as a positive control. The gel was stained with ethidium bromide to visualise DNA bands. Obtained images were exported to BioNumerics software ver. 7.6. (Applied Maths). PFGE patterns were compared using the Dice similarity coefficient. A dendrogram showing genetic relatedness of the strains was constructed by the single linkage method with $1 \%$ tolerance and $1 \%$ optimisation of band position. PFGE fingerprints with $\geq 85 \%$ similarity were categorised as clonally related.

\section{Results}

Population of $P$. aeruginosa strains

A clonal analysis of $P$. aeruginosa strains was carried out for 202 strains isolated from 11 different hospitals located in north-western Poland. Pseudomonas aeruginosa isolates were distributed among 116 different pulsotype groups. Thirty clusters consisted of two or more strains. The clusters were assigned with different letter designations from A to AH. A total of 86 clusters contained only one strain. Strains clonally related with at least one other isolate in this study were found in all hospitals except hospital H6. Five groups of clonally related strains (D, E, J, T, AC) included more than 5 strains (Fig. 1). The most prevalent $\mathrm{D}$ cluster included 17 strains isolated from two hospitals $\mathrm{H} 2(n=16)$ and $\mathrm{H} 1 \quad(n=1)$. Strains of those groups were isolated at the ICU $(n=7)$, $\mathrm{BU}(n=8)$, and surgical unit (SU, $n=1)$, from December 2015 to March 2017. All isolates of group D were resistant to imipenem. Group E included 13 isolates collected between December 2015 and December 2016. Isolates of this cluster were isolated from different patients of an ICU $(n=12)$ and orthopaedic unit $(n=1)$ of hospital H3. Isolates of group D and E represented 8.6 and $6.4 \%$ of all isolates collected, respectively. Strains of cluster E were isolated 12 times from patients at ICUs of hospital H3, which stands for $25 \%(12 / 48)$ of all isolates collected in this hospital. Strains of cluster D were collected 16 times from patients of a burn unit and the ICU of $\mathrm{H} 2$, 


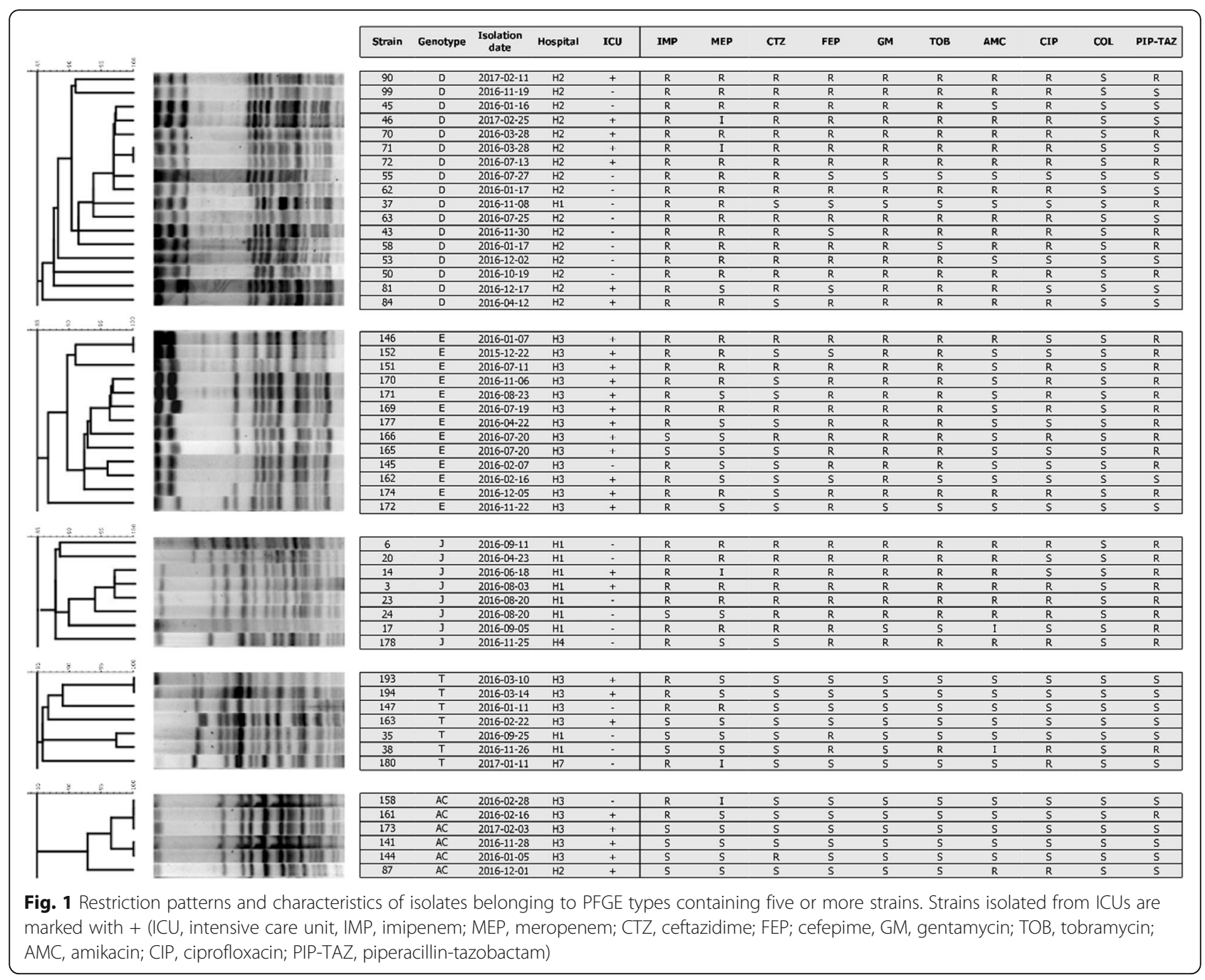

representing $25.4 \%$ of all isolates from this medical centre. Most of the isolates of cluster D were resistant to carbapenems (14/17), aminoglycosides (12/17), and other antimicrobials tested except piperacillin with tazobactam and colistin. Similar high rates of resistance to various antimicrobial classes were noted for strains from pulsotype E. Both clusters D and E share resistance to imipenem and aminoglycosides.

Strains of pulsotypes J, T, and AC were isolated 8, 7 and 6 times from different patients. Restriction patterns, hospitals, wards, isolation dates, and antimicrobial susceptibility patterns of $P$. aeruginosa strains isolates belonging to the major pulsotype groups are shown in Fig. 1. In this study, we found 13 clusters of strains: A, D, J, K, M, N, O, P, T, X, AC, AD, and $\mathrm{AH}$ that were isolated from patients of two or more hospitals. Strains of group T were collected in 3 different hospitals $\mathrm{H} 1 \quad(n=2), \mathrm{H} 3(n=4)$, and $\mathrm{H} 7(n=1)$. Isolates of the $\mathrm{T}$ cluster were susceptible to most of the antimicrobials tested, except for imipenem, for which 4 strains were resistant.

Most of the $P$. aeruginosa strains from this study were isolated in ICUs $(n=81)$. All remaining isolates were isolated in $\mathrm{BU}(n=35), \mathrm{SU}(n=35)$, and other units $(n=$ $51)$. Strains from clusters containing two and more strains were most frequently isolated in ICUs. Among strains isolated from these units, 56/81 (69.1\%) were clonally related to at least one other strain in this study. In BU, 21/35 (60\%) isolates have similar restriction patterns shared by two or more strains. In Fig. 2, we demonstrated a distribution of PFGE clusters of various sizes among medical units of different types.

Overall, our findings show the presence of multiple $P$. aeruginosa pulsotypes and a constant exchange of strains inside a single hospital ward, between different hospital wards and between different hospitals. Part of the clonally related strains were constantly isolated from different patients of the same hospital ward. Results presented 


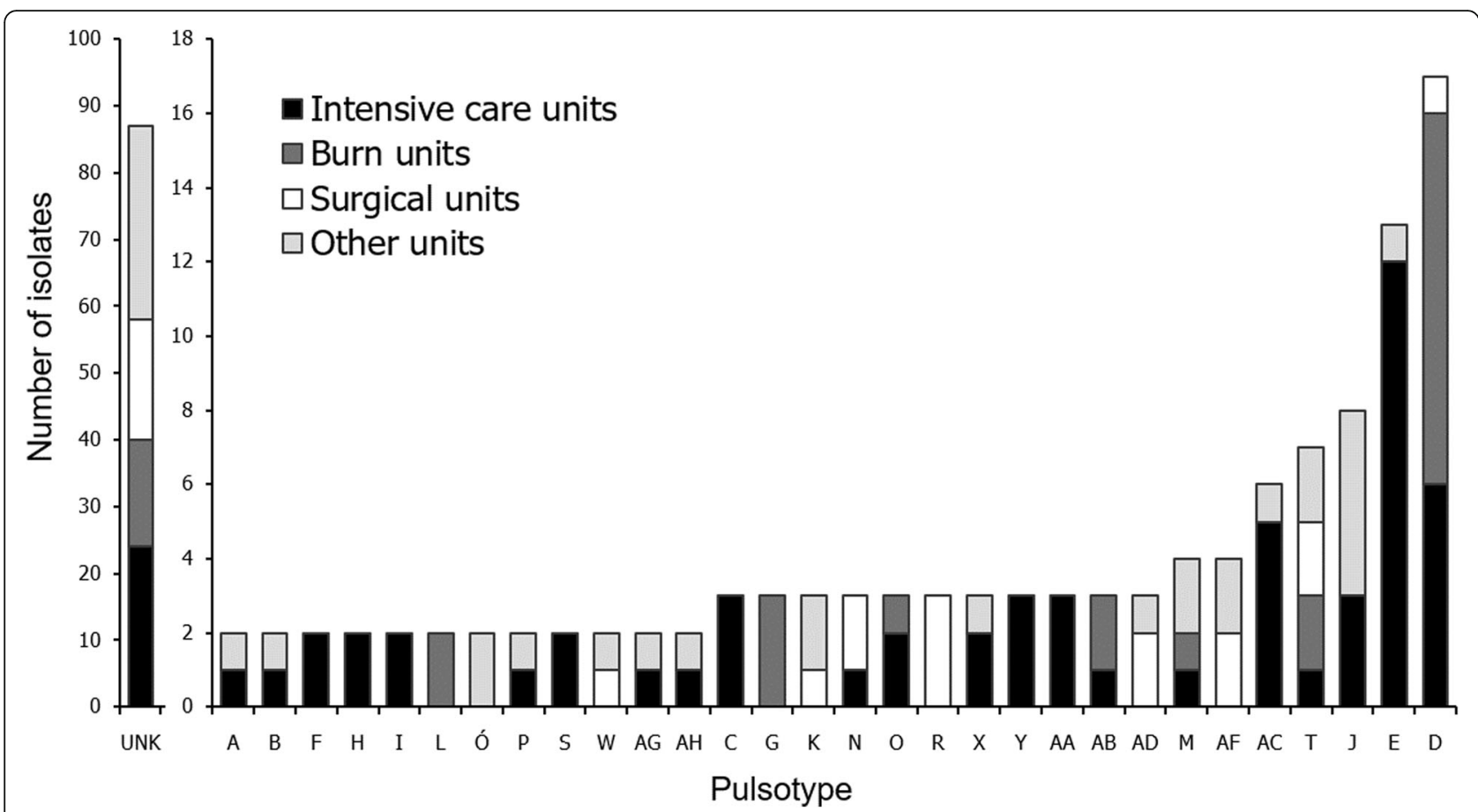

Fig. 2 Distribution of isolates belonging to different PFGE types among intensive care units, burn units, surgical units, and other units (UNK, unique pulsotype)

in this work suggest a non-clonal structure of $P$. aeruginosa population of strains isolated at hospital centres located in north-western Poland.

\section{Antimicrobial resistance prevalence}

Resistance to tested antipseudomonal drugs was as follows (202 strains): imipenem 67.8\% $(n=137)$, meropenem $29.2 \%(n=59)$, ceftazidime $33.2 \%(n=67)$, cefepime $42.6 \%$ $(n=86)$, piperacillin-tazobactam $39.6 \%(n=80)$, gentamicin $37.6 \%(n=76)$, amikacin $30.2 \%(n=61)$, tobramycin $38.1 \%(n=77)$, ciprofloxacin $39.6 \%(n=80)$, and colistin $3.0 \%(n=6)$. The prevalence of resistance to both carbapenems was $29.2 \%$, and for all aminoglycosides $22.8 \%$. Combined resistance to carbapenems and aminoglycosides was $14.4 \%(n=29)$. Out of 202 isolates in this study, $48 \%(n=97)$ were multidrug-resistant (MDR). Multidrugresistance was defined as not susceptible to 3 or more antibiotic classes $(n=20)$ [22].

Among MDR strains, $71.1 \%(n=69)$ of isolates were clonally related with at least one more isolate in the study. However non-MDR susceptible strains $(n=105)$ less frequently exhibited clonal relatedness with other strains $44.8 \%(n=47)$.

We observed a variation in antimicrobial resistance rates between different hospital wards. Strains isolated from BU were most often resistant to the tested antimicrobials except piperacillin with tazobactam, ciprofloxacin and colistin. Data regarding antimicrobial resistance of strains isolated at different medical units are shown in Table 2.

\section{Discussion}

This study allows for a description of the population structure of $P$. aeruginosa clinical isolates collected from hospitals of north-western Poland. It revealed the presence of 30 clusters of related strains and 86 unique strains. The obtained results indicate a predominantly non-clonal population structure and continuous exchange of $P$. aeruginosa strains between patients of the same and different hospital wards, and even between different hospitals in north-western Poland. Similar results have been presented in the previous epidemiological study conducted in this region [23]. Authors indicated a frequent transmission of $P$. aeruginosa strains between patients in medical centres. Our results are also in concordance with other epidemiological studies demonstrating a non-clonal population structure of $P$. aeruginosa $[12,15,16]$. It has been reported that the relatively high recombination frequency of $P$. aeruginosa strains is considered to be a key driver of the multiclonal population structure $[12,13]$.

Our results indicate continuous isolation of clonally related $P$. aeruginosa pathogens from different patients on the same ward. Hospital staff were not aware about the spread of pathogens during that time and did not 
Table 2 Antimicrobial resistance rates of strains isolated from patients of different medical wards (ICU, intensive care unit; BU, burn unit; SU, surgical unit; OU, other units; $n$, number of isolates; \%R, resistance rate; $95 \% \mathrm{Cl}, 95 \%$ confidence interval)

\begin{tabular}{|c|c|c|c|c|c|c|c|c|c|c|}
\hline \multirow[t]{2}{*}{ Antimicrobial agent } & \multicolumn{2}{|c|}{ ICU $(n=81)$} & \multicolumn{2}{|c|}{$\mathrm{BU}(n=35)$} & \multicolumn{2}{|c|}{ SU $(n=32)$} & \multicolumn{2}{|c|}{ OU $(n=54)$} & \multicolumn{2}{|c|}{ All units $(n=202)$} \\
\hline & $\% R$ & $95 \% \mathrm{Cl}$ & $\% R$ & $95 \% \mathrm{Cl}$ & $\% R$ & $95 \% \mathrm{Cl}$ & $\mathrm{R} \%$ & $95 \% \mathrm{Cl}$ & $\mathrm{R} \%$ & $95 \% \mathrm{Cl}$ \\
\hline Imipenem & 71.1 & 9.6 & 72.7 & 15.2 & 58.3 & 19.7 & 65.5 & 12.6 & 67.8 & 6.4 \\
\hline Meropenem & 28.9 & 9.6 & 51.5 & 17.1 & 20.8 & 16.2 & 21.8 & 10.9 & 29.2 & 6.3 \\
\hline Ceftazidim & 32.5 & 9.9 & 51.5 & 17.1 & 37.5 & 19.4 & 23.6 & 11.2 & 33.2 & 6.5 \\
\hline Cefepime & 43.4 & 10.5 & 48.5 & 17.1 & 41.7 & 19.7 & 38.2 & 12.8 & 42.6 & 6.8 \\
\hline Piperacillin/tazobactam & 48.2 & 10.6 & 24.2 & 14.6 & 37.5 & 19.4 & 38.2 & 12.8 & 39.6 & 6.7 \\
\hline Gentamicin & 33.7 & 10 & 57.6 & 16.9 & 33.3 & 18.9 & 34.5 & 12.6 & 37.6 & 6,7 \\
\hline Tobramycin & 31.3 & 9.8 & 57.7 & 16.9 & 45.8 & 19.9 & 34.5 & 12.6 & 38.1 & 6.7 \\
\hline Amikacin & 19.3 & 8.3 & 57.8 & 16.9 & 33.3 & 18.9 & 30.9 & 12.2 & 30.7 & 6.4 \\
\hline Ciprofloxacin & 28.9 & 9.6 & 51.5 & 17.1 & 66.7 & 18.9 & 34.5 & 12.6 & 39.1 & 6.4 \\
\hline Colistin & 1.2 & 2.3 & 0 & - & 8.3 & 11.0 & 5.5 & 6.0 & 3.0 & 2.4 \\
\hline
\end{tabular}

report any outbreak. The two largest PFGE types, D and $E$, were described as endemic. Strains of those groups were isolated 17 and 13 times in hospital wards during a period of 1 year; however, no increase in infection rates was reported by the hospital staff. Isolates of cluster $\mathrm{E}$ were isolated 12 times from patients at ICUs of a hospital in Szczecin (H3), which stands for 25\% of all isolates from that hospital. Strains of cluster D represented $25.4 \%$ of all isolates from the burn unit and ICUs of a hospital in Gryfice (H2) (Fig. 2). In other studies, the most prevalent PFGE types were shared by $22-52 \%$ of all $P$. aeruginosa isolates in a single hospital or medical unit [24-26]. Strains in these studies were continuously isolated from patients of hospital units and were described as endemic. Due to the hospital selection pressure, the resistance of endemic strains to multiple antimicrobial agents appears to be the determining factor of their endemicity [27].

Patients of intensive care and burn units are particularly at risk of nosocomial infections caused by $P$. aeruginosa [6, 18]. The results of this work demonstrate that the transmission of $P$. aeruginosa strains was most frequent in intensive care and burn units. Endemic strains of clusters D and E were also mostly isolated from patients of medical wards of these types.

In other medical units, the transmission of strains between patients was also possible, however, less frequent. The risk of colonisation by $P$. aeruginosa hospital strains at ICUs is higher presumably due to the prolonged stay of patients, the severity of their illness and exposure to invasive medical procedures [28]. Detection of strains disseminated in hospital wards seems critically important for immunocompromised patients and other patients susceptible to $P$. aeruginosa infections.

In this study clonally related strains were also isolated from patients of two or more hospitals, which indicates spread of these pathogens between medical centres in north-western Poland. It is thought that related strains can be transferred between different hospitals via hospital staff or their residents [29].

Epidemic and endemic strains are often multiple drug resistant which is responsible for the increased mortality of those patients. Inappropriate empirical therapy is perceived as the main factor contributing to increased mortality [30]. This study, in a similar way, demonstrates that MDR strains were more frequently found in clusters of clonally related strains. Among MDR $P$. aeruginosa strains, $71.1 \%$ exhibited clonal relatedness with at least one other strain. Strains of cluster D and E were resistant to imipenem and aminoglycosides. Resistance to other antimicrobials was also common in these clusters.

Detection and elimination of dissemination of highrisk clones in many cases is not possible with the use of simple epidemiological data alone $[12,31]$. This is also the case in this study, where we demonstrated the presence of endemic strains at ICU and burn units that were not detected by hospital staff. Epidemiological studies with the use of molecular methods were not conducted at these wards at the time when clonally related strains were isolated. Thus, the use of molecular typing methods is necessary, especially at various ICU and BU, to establish a possible transmission of clonally related strains between patients that may appear in the future. The establishment of new infection prevention and control strategies should also be considered.

The obtained data regarding antimicrobial resistance indicate that levels of resistance of $P$. aeruginosa strains isolated from north-western Poland are comparable with results for the whole country [32]. According to a 2017 ECDC survey, the average resistance rate to carbapenems (imipenem+meropenem) was $24.2 \%$ across Poland, whereas in this research, $28.7 \%$ of strains were resistant to carbapenems. Discrepancies between results can be 
due to different infection control management in hospitals, misuse of antimicrobial agents, sanitation and distributions of strains in the region [33].

The lowest resistance rate among used aminoglycosides has been observed for amikacin. Relatively lower amikacin resistance rates of $P$. aeruginosa strains were also noted in other studies. Sader et al. [34] compared resistance rates of $P$. aeruginosa strains originating from multiple medical centres in the USA, and recorded susceptibility rates to gentamicin, tobramycin, and amikacin of 88,90 , and $98 \%$, respectively. Similar data was obtained from research conducted in China, where amikacin was the second (after colistin) most effective antibiotic [35]. Lower amikacin resistance rates comparing to resistance to other aminoglycosides was also observed for multi-drug resistant $P$. aeruginosa strains [36]. Results presented in this and other epidemiological studies suggest that the development of amikacin resistance is less common than for other aminoglycosides in P. aeruginosa strains, and therefore that the use of amikacin could provide a better chance of success in empiric therapy. The lowest resistance rate indicated was that for colistin $(\sim 3 \%)$. Previously reported colistin resistance rates among various $P$. aeruginosa strains worldwide varied between 0 to $36 \%[37,38]$. Colistin is used to treat $P$. aeruginosa infection due to the MDR profiles of many strains, which mean alternative antibiotics cannot be prescribed. However, colistin is not used routinely due to its diverse side effects, including neuro- and nephrotoxicity [39].

This work has certain limitations. We acknowledge that the collected strains represented only a part of $P$. aeruginosa strains isolated from patients at hospitals in north-western Poland. We cannot assure that some unique strains were not in fact clonally related with other strains from other medical centres. However, this is the largest $P$. aeruginosa genotyping study that was ever performed in the region of north-western Poland. The investigated amount of strains allowed us to demonstrate a non-clonal population structure and reveal the presence of endemic $P$. aeruginosa strains in hospitals of the selected region. Secondly, this analysis relied on clinical $P$. aeruginosa strains collected only from patients. Environmental samples were not collected for this study. Additional strains from nosocomial environments could help us determine the possible hospital sources of $P$. aeruginosa strains in hospitals. Furthermore, it would be interesting to look into the mechanisms of resistance of multidrug-resistant strains, especially clonally related strains that were spreading over the ICU and BU. Metallo- $\beta$-lactamase (MBL) producers are commonly found in hospitals of Western-Europe, and are frequently responsible for outbreaks in ICU units [40-42]. Therefore it would be interesting to know the proportion of MBL producers in our hospitals.

The use of PFGE method for genotyping could also be considered as a drawback of this study. It is timeconsuming, relatively expensive, and the results are difficult to compare interlaboratory [43]. Additional implementation of other typing methods would have additional value to the study. Performing MLST would allow us to compare results internationally.

However, there are a number of genotyping studies in which PFGE is still used [44, 45]. Although this method was first introduced in 1986, it is still frequently used for typing clinically relevant microorganisms.

\section{Conclusions}

The results of this study show a constant exchange of different $P$. aeruginosa isolates between patients of medical wards in the same wards, different wards and even different hospitals of Poland. A high number of different PFGE types indicates a non-clonal population structure of $P$. aeruginosa species. These results are in congruence with previous similar studies. The results indicate the absence of a $P$. aeruginosa outbreak in Poland, although some endemic strains are undoubtedly present in intensive care and burn units. An antimicrobial resistance analysis showed that the antimicrobial resistance rates of $P$. aeruginosa isolates from hospitals of the selected region are comparable with the results from other parts of Poland. The revealed prolonged isolation of highly resistant endemic strains in hospitals indicates that more frequent use of genotyping methods in hospital wards, especially in intensive care and burn units, should be considered in the future.

\section{Abbreviations}

ICU: Intensive care unit; BU: Burn unit; SU: Surgical unit; OU: Other units; n: Number of isolates; H1: Multispeciality Voivodeship Hospital in Gorzów Wielkopolski; H2: Regional Specialist Hospital in Gryfice; H3: Independent Public Clinical Hospital no. 1, Pomeranian Medical University in Szczecin; H4: Independent Public Clinical Hospital no. 2, Pomeranian Medical University in Szczecin; H5: Specialised Zdroje Hospital in Szczecin; H6: Sokolowski Specialist Hospital; H7: Hospital of the Ministry of the Interior and Administration in Szczecin; H8: West Pomeranian Oncology Centre in Szczecin; H9: West Pomeranian Hospice for Children in Szczecin;

H10: Independent Public Clinical Hospital no. 1, Pomeranian Medical University; H11: Residential Health Care Centre in Resko

\section{Acknowledgements}

We would like to thank the microbiology laboratories of the hospitals in north-western Poland, for supplying the $P$. aeruginosa clinical isolates. We thank to Danuta Michałek-Aniko, Grażyna Krawczyńska, Katarzyna Kopron, Elżbieta Justyńska, Iwona Bilska, Adam Smolak, Beata Walaszkowska for their support to collect bacterial isolates.

Authors' contributions

$M B$, JJK and DKB contributed to the study conception and design. Material preparation and data collection was performed by MB and JJK. Analysis was performed by MB, ŻK and KG. The draft of the manuscript was written by 
MB, JJK and DKB, and all authors commented on previous versions of the manuscript. All authors approved the final manuscript.

\section{Funding}

This study was supported by the Pomeranian Medical University Research Program for Young Scientists, grant no. MB-134-211/17 (M.B. and WFB-430$01 / S / 14 / 2020$ ) The funder had a role in the collection and analysis of data.

\section{Availability of data and materials}

The data sets used and/or analyzed during the current study are available from the corresponding author on reasonable request.

\section{Ethics approval and consent to participate}

Not applicable. In the study, informed consent was not required as the isolates included in the study were obtained as a result of standard medical care. Written permissions to access the raw data and samples mentioned in the methods were obtained from hospitals authorities. Patient identity, as well as all personal information, remained confidential.

\section{Consent for publication}

Not applicable.

\section{Competing interests}

The authors declare that they have no competing interests.

\section{Author details}

${ }^{1}$ Department of Medical Microbiology, Chair of Microbiology, Immunology and Laboratory Medicine, Pomeranian Medical University in Szczecin, Powstanców Wielkopolskich 72, 70-111 Szczecin, Poland. ²Department of Laboratory Medicine; Chair of Microbiology, Immunology and Laboratory Medicine, Pomeranian Medical University in Szczecin, Powstanców Wielkopolskich 72, 70-111 Szczecin, Poland. ${ }^{3}$ Independent of Pharmaceutical Botany, Pomeranian Medical University in Szczecin, Powstanców Wielkopolskich 72, 70-111 Szczecin, Poland

Received: 18 March 2020 Accepted: 8 September 2020

Published online: 22 September 2020

\section{References}

1. Vincent $J L$, Rello J, Marshall J, et al. International study of the prevalence and outcomes of infection in intensive care units. JAMA. 2009;302:2323-9.

2. Livermore DM. Multiple mechanisms of antimicrobial resistance in Pseudomonas aeruginosa: our worst nightmare? Clin Infect Dis. 2002;34:634-40.

3. Muscarella LF. Contribution of tap water and environmental surfaces to nosocomial transmission of antibiotic-resistant Pseudomonas aeruginosa. Infect Control Hosp Epidemiol. 2004;25:342-5.

4. Gómez-Zorrilla S, Camoez M, Tubau F, Cañizares R, Periche E, Dominguez MA, Ariza J, Peña C. Prospective observational study of prior recta colonization status as a predictor for subsequent development of Pseudomonas aeruginosa clinical infections. Antimicrob Agents Chemother. 2015;59:5213-9.

5. D'Agata E. Pseudomonas aeruginosa and other Pseudomonas species. In: Bennet JE, Dolin R, Blaser M, editors. Principles and practice of infectious diseases. 8th ed. Philadelphia: Elsevier; 2015. p. 2518-32.

6. Blanc DS, Francioli P, Zanetti G. Molecular epidemiology of Pseudomonas aeruginosa in the intensive care units - a review. Open Microbiol J. 2007;1: 8-11.

7. Bicking Kinsey C, Koirala S, Solomon B, Rosenberg J, Robinson BF, Neri A, Laufer Halpin A, Arduino MJ, Moulton-Meissner H, Noble-Wang J, Chea N, Gould CV. Pseudomonas aeruginosa outbreak in a neonatal intensive care unit attributed to hospital tap water. Infect Control Hosp Epidemiol. 2017; 38:801-8.

8. Vianelli N, Giannini MB, Quarti C, Bucci Sabattini MA, Fiacchini M, de Vivo A, Graldi P, Galli S, Nanetti A, Baccarani M, Ricci P. Resolution of a Pseudomonas aeruginosa outbreak in a hematology unit with the use of disposable sterile water filters. Haematologica. 2006;91:983-5.

9. European Centre for Disease Prevention and Control (2017) Healthcareassociated infections in intensive care units - Annual Epidemiological Report for 2015. https://www.ecdc.europa.eu/en/publications-data/healthcareassociated-infections-intensive-care-units-annual-epidemiological. Accessed 03 June 2019
10. Kayabas U, Bayraktar M, Otlu B, Ugras M, Ersoy Y, Bayindir Y, Durmaz R. An outbreak of Pseudomonas aeruginosa because of inadequate disinfection procedures in a urology unit: a pulsed-field gel electrophoresis-based epidemiologic study. Am J Infect Control. 2008;36:33-8.

11. Weber DJ, Rutala WA, Sickbert-Bennett EE. Outbreaks associated with contaminated antiseptics and disinfectants. Antimicrob Agents Chemother. 2007;51:4217-24.

12. Maatallah M, Cheriaa J, Backhrouf $A$, Iversen $A$, Grundmann H, Do T, Do T, Lanotte P, Mastouri M, Elghmati MS, Rojo F, Mejdi S, Giske CG. Population structure of Pseudomonas aeruginosa from five Mediterranean countries: evidence for frequent recombination and epidemic occurrence of CC235. PLoS One. 2011;6:e25617.

13. Kidd TJ, Ritchie SR, Ramsay KA, Grimwood K, Bell SC, Rainey PB. Pseudomonas aeruginosa exhibits frequent recombination, but only a limited association between genotype and ecological setting. PLoS One 2012;7:e44199.

14. Darch SE, Simoska O, Fitzpatrick M, Barraza JP, Stevenson KJ, Bonnecaze RT, Shear JB, Whiteley M. Spatial determinants of quorum signaling in a Pseudomonas aeruginosa infection model. Proc Natl Acad Sci U S A. 2018; 115:4779-84.

15. Pirnay JP, Biloca F, Pot B, Cornelis P, Zizi M, Van Eldere J, Deschaght $P$, Vaneechoutte M, Jennes S, Pitt T, De Vos D. Pseudomonas aeruginosa population structure revisited. PLoS One. 2009;4:e7740.

16. McCarthy K. Pseudomonas aeruginosa: evolution of antimicrobial resistance and implications for therapy. Semin Respir Crit Care Med. 2015;36:044-55.

17. Bukholm G, Tannaes T, Kjelsberg AB, Smith-Erichsen N. An outbreak of multidrug-resistant Pseudomonas aeruginosa associated with increased risk of patient death in an intensive care unit. Infect Control Hosp Epidemiol. 2002;23:441-6

18. Decraene V, Ghebrehewet S, Dardamissis E, Huyton R, Mortimer K, Wilkinson D, Shokrollahi K, Singleton S, Patel B, Turton J, Hoffman P, Puleston R. An outbreak of multidrug-resistant Pseudomonas aeruginosa in a burns service in the north of England: challenges of infection prevention and control in a complex setting. J Hosp Infect. 2018;100:e239-45.

19. Durojaiye OC, Carbarns N, Murray S, Majumdar S. Outbreak of multidrugresistant Pseudomonas aeruginosa in an intensive care unit. J Hosp Infect. 2011;78:154-5

20. Spilker T, Coenye T, Vandamme P, LiPuma JJ. PCR-based assay for differentiation of Pseudomonas aeruginosa from other Pseudomonas species recovered from cystic fibrosis patients. J Clin Microbiol. 2004:42:2074-9.

21. The European Committee on Antimicrobial Susceptibility Testing. Clinical breakpoints and dosing of antibiotics. http://www.eucast.org/clinical_ breakpoints. Accessed 03 June 2019.

22. Magiorakos AP, Srinivasan A, Carey RB, Carmeli Y, Falagas ME, Giske CG, Harbart S, Hindler JF, Kahlmeter G, Olsson-Liljequist B, Paterson DL, Rice LB, Stelling J, Struelens MJ, Vatopoulos A, Weber JT, Monnet DL. Multidrugresistant, extensively drug-resistant and pandrug-resistant bacteria: an international expert proposal for interim standard definitions for acquired resistance. Clin Microbiol Infect. 2012;18:268-81.

23. Czekajło-Kołodziej U, Giedrys-Kalemba S, Medrala D. Phenotypic and genotypic characteristics of Pseudomonas aeruginosa strains isolated from hospitals in the north-west region of Poland. Pol J Microbiol. 2006;55:103-12.

24. Quick J, Cumley N, Wearn CM, Niebel M, Constantinidou C, Thomas CM Pallen MJ, Moiemen NS, Bamford A, Oppenheim B, Loman NJ. Seeking the source of Pseudomonas aeruginosa infections in a recently opened hospital: an observational study using whole-genome sequencing. BMJ Open. 2014;4 e006278.

25. Tassios PT, Gennimata V, Spaliara-Kalogeropoulou L, Kairis D, Koutsia C, Vatopoulos AC, Legakis NJ. Multiresistant Pseudomonas aeruginosa serogroup 0:11 outbreak in an intensive care unit. Clin Microbiol Infect. 1997;3:621-8.

26. Telling K, Laht M, Brauer A, Remm M, Kisand V, Maimets M, Tenson T, Lutsar I. Multidrug resistant Pseudomonas aeruginosa in Estonian hospitals. BMC Infect Dis. 2018;18:513

27. Alcántar-Curiel MD, Ledezma-Escalante CA, Jarillo-Quijada MD, GayossoVázquez C, Morfín-Otero R, Rodríguez-Noriega E, Cedillo-Ramírez ML, Santos-Preciado Jl, Girón JA. Association of antibiotic resistance, cell adherence, and biofilm production with the endemicity of nosocomial Klebsiella pneumoniae. Biomed Res Int. 2018;2018:7012958.

28. Shi Q, Huang C, Xiao T, Wu Z, Xiao Y. A retrospective analysis of Pseudomonas aeruginosa bloodstream infections: prevalence, risk factors, 
and outcome in carbapenem-susceptible and -non-susceptible infections. Antimicrob Resist Infect Control. 2019;8:68.

29. Fujimura S, Nakano Y, Takane H, Kikuchi T, Watanabe A. Risk factors for health care-associated pneumonia: transmission of multidrug-resistant Pseudomonas aeruginosa isolates from general hospitals to nursing homes. Am J Infect Control. 2011;39(2):173-5.

30. Dantas RC, Ferreira ML, Gontijo-Filho PP, Ribas RM. Pseudomonas aeruginosa bacteraemia: independent risk factors for mortality and impact of resistance on outcome. J Med Microbiol. 2014;63:1679-87.

31. Singh A, Goering RV, Simjee S, Foley SL, Zervos MJ. Application of molecular techniques to the study of hospital infection. Clin Microbiol Rev. 2006;19: $512-30$.

32. European Centre for Disease Prevention and Control. Surveillance of antimicrobial resistance in Europe 2017. https://www.ecdc.europa.eu/en/ publications-data/surveillance-antimicrobial-resistance-europe-2017. Accessed 03 June 2019.

33. Farajzadeh Sheikh A, Shahin M, Shokoohizadeh L, Halaji M, Shahcheraghi F, Ghanbari F. Molecular epidemiology of colistin-resistant Pseudomonas aeruginosa producing NDM-1 from hospitalized patients in Iran. Iran J Basic Med Sci. 2019;22:38-42.

34. Sader HS, Castanheira M, Shortridge D, Mendes RE, Flamm RK. Antimicrobial activity of Ceftazidime-Avibactam tested against multidrug-resistant Enterobacteriaceae and Pseudomonas aeruginosa isolates from U.S. medical centers, 2013 to 2016. Antimicrob Agents Chemother. 2017;61:e01045-17.

35. Fan X, Wu Y, Xiao M, Xu ZP, Kudinha T, Bazaj A, Kong F, Xu YC. Diverse genetic background of multidrug-resistant Pseudomonas aeruginosa from mainland China, and emergence of an extensively drug-resistant ST292 clone in Kunming. Sci Rep. 2016;6:26522.

36. Gallagher JC, Satlin MJ, Elabor A, Saraiya N, McCreary EK, Molnar E, ElBeyrouty C, Jones BM, Dixit D, Heil EL, Claeys KC, Hiles J, Vyas NM, Bland CM, Suh J, Biason K, McCoy D, King MA, Richards L, Harrington N, Guo Y, Chaudhry S, Lu X, Yu D. Ceftolozane-Tazobactam for the Treatment of Multidrug-Resistant Pseudomonas aeruginosa Infections: A Multicenter Study. Open Forum Infect Dis. 2018;5:ofy280

37. Maroui I, Barguigua A, Aboulkacem A, Ouarrak K, Sbiti M, Louzi H, Timinouni M, Belhaj A. First report of VIM-2 metallo- $\beta$-lactamases producing Pseudomonas aeruginosa isolates in Morocco. J Infect Chemother. 2015;22: 127-32.

38. Pedersen MG, Olesen HV, Jensen-Fangel S, Nørskov-Lauritsen N, Wang M. Colistin resistance in Pseudomonas aeruginosa and Achromobacter spp. cultured from Danish cystic fibrosis patients is not related to plasmidmediated expression of mcr-1. J Cyst Fibros. 2018;17:e22-3.

39. Martis N, Leroy S, Blanc V. Colistin in multi-drug resistant Pseudomonas aeruginosa blood-stream infections: a narrative review for the clinician. J Inf Secur. 2014;69:1-12.

40. Fiett J, Baraniak A, Mrówka A, Fleischer M, Drulis-Kawa Z, Naumiuk Ł, Samet A, Hryniewicz W, Gniadkowski M. Molecular epidemiology of acquiredmetallo-beta-lactamase-producing bacteria in Poland. Antimicrob Agents Chemother. 2006:50:880-6.

41. Lepsanovic Z, Libisch B, Tomanovic B, Nonkovici Z, Balogh B, Füzi M. Characterisation of the first VIM metallo-beta-lactamase-producing Pseudomonas aeruginosa clinical isolate in Serbia. Acta Microbiol Immunol Hung. 2008;55:447-54.

42. Timofte D, Panzaru CV, Maciuca IE, Dan M, Mare AD, Man A, Toma F. Active surveillance scheme in three Romanian hospitals reveals a high prevalence and variety of carbapenamase-producing Gram-negative bacteria: a pilot study. Euro Surveill. 2016;21. https://doi.org/10.2807/1560-7917.ES.2016.21.25. 30262.

43. Top J, Banga NM, Hayes R, Willems RJ, Bonten MJ, Hayden MK. Comparison of multiple-locus variable-number tandem repeat analysis and pulsed-field gel electrophoresis in a setting of polyclonal endemicity of vancomycinresistant Enterococcus faecium. Clin Microbiol Infect. 2008;14:363-9.

44. Cigana C, Melotti P, Baldan R, Pedretti E, Pintani E, lansa P, De Fino I, Favari F, Bergamini G, Tridello G, Cirillo DM, Assael BM, Bragonzi A. Genotypic and phenotypic relatedness of Pseudomonas aeruginosa isolates among the major cystic fibrosis patient cohort in Italy. BMC Microbiol. 2016;16:142.

45. Parcell BJ, Oravcova K, Pinheiro M, Holden MTG, Phillips G, Turton JF, Gillespie SH. Pseudomonas aeruginosa intensive care unit outbreak: winnowing of transmissions with molecular and genomic typing. J Hosp Infect. 2018;98:282-8.

\section{Publisher's Note}

Springer Nature remains neutral with regard to jurisdictional claims in published maps and institutional affiliations.

\section{Ready to submit your research? Choose BMC and benefit from:}

- fast, convenient online submission

- thorough peer review by experienced researchers in your field

- rapid publication on acceptance

- support for research data, including large and complex data types

- gold Open Access which fosters wider collaboration and increased citations

- maximum visibility for your research: over $100 \mathrm{M}$ website views per year

At $\mathrm{BMC}$, research is always in progress.

Learn more biomedcentral.com/submissions 